\title{
Risk factors for early angle-closure disease in a Burmese population: the Meiktila Eye Study
}

RJ Casson', D Marshall', HS Newland', S McGovern'1, J Muecke', EWH Tan'1, D Selva' and T Aung ${ }^{2}$

\begin{abstract}
Purpose To determine risk factors for early angle-closure disease (AD) in a Burmese population.
\end{abstract}

Methods A cross-sectional, population-based survey of the inhabitants, 40 years of age and over, from villages in the Meiktila District was performed; 2481 eligible participants were identified, 2076 participated in the study, and 2050 could be categorized as having early AD (defined by the presence of so-called occludable angles ( $<90$ degrees of posterior trabecular meshwork visible on gonioscopy), but without glaucomatous optic neuropathy). The ophthalmic examination included Snellen visual acuity, slit-lamp examination, tonometry, gonioscopy, biometry, and dilated stereoscopic fundus examination.

Results The mean axial length (AL) and anterior chamber depth (ACD) in those with occludable angles were 21.9 and $2.60 \mathrm{~mm}$, respectively; in those with non-occludable angles, the corresponding lengths were 22.74 and $2.84 \mathrm{~mm}$, respectively $(P<0.001$ for both comparisons). In the univariate analyses, age, female gender, nuclear and cortical cataract, steeper corneal curvature, more anterior lens position, and myopia were also significantly associated with occludability. In the multivariate analysis, age, $\mathrm{AL}, \mathrm{ACD}$, and nuclear cataract were significantly associated with occludability.

Conclusion In this Burmese population, those with occludable angles had significantly shorter ALs, ACDs, and thicker lenses than those without occludable angles. In multivariate analysis, increasing age, decreasing $\mathrm{AL}$, decreasing $\mathrm{ACD}$, and nuclear cataract were significant predictors of early AD. The presence of nuclear cataract per se should raise clinical suspicion of the possibility of AD in this population. Eye (2009) 23, 933-939; doi:10.1038/eye.2008.102; published online 18 April 2008

Keywords: angle-closure glaucoma; ocular biometry; nuclear cataract

\section{Introduction}

Glaucoma is the second most common cause of blindness worldwide, and the majority of those blinded resides in Asia. ${ }^{1}$ It is well recognized that primary angle closure glaucoma (PACG) is more common in people of Asian ethnicity than those with European or African ethnicity, ${ }^{2-4}$ and because of its devastating effect on vision, accounts for about half of the world's glaucoma blindness. ${ }^{1}$ Other demographic risk factors for PACG include increasing age and female gender. ${ }^{5,6}$ In addition, PACG is traditionally associated with ocular biometric features that produce a 'crowded anterior segment', including short axial length (AL), shallow anterior chamber depth (ACD), and increased lens thickness. ${ }^{6}$ A shallow ACD is a significant risk factor for angle closure in Mongolians ${ }^{7}$ and Chinese Singaporeans. ${ }^{8}$ However, the ACD in Taiwanese Chinese eyes is similar to that in Caucasians, ${ }^{9}$ suggesting existence of as yet unidentified risk factors for PACG in Asian eyes.

PACG is the last stage of a disease process that begins with an 'at risk' eye. In the early stages of the disease, the iridocorneal angle is narrow and there is reversible occlusion of the trabecular meshwork (TM) by the peripheral iris (appositional closure). With progression, this occlusion becomes irreversible and aqueous outflow is compromised (synechial closure); the intraocular pressure (IOP) elevates and glaucomatous optic neuropathy may ensue. Appositional and synechial angle closure are
${ }^{1}$ South Australian Institute of Ophthalmology, Department of Ophthalmology and Visual Sciences, University of Adelaide, Adelaide, South Australia, Australia

${ }^{2}$ Yangon Eye Hospital, Yangon, Myanmar

Correspondence: RJ Casson Associate Professor, South Australian Institute of Ophthalmology,

Department of

Ophthalmology and Visual Sciences,

University of Adelaide, South Australia 5000, Australia

Tel: + 61882222729

Fax: + 61882222741

E-mail: robert.casson@ adelaide.edu.au

Received: 24 August 2007 Accepted in revised form: 8 March 2008 Published online: 18 April 2008 
considered to represent early stages in the natural history of angle-closure disease (AD) $)^{5,10}$ and reflect the long-term risk of visual morbidity. ${ }^{11}$

We recently conducted a population-based eye study in central Myanmar (formerly Burma). PACG accounted for $84 \%$ of all glaucoma blindness in this region, with the majority due to acute angle-closure glaucoma

(AACG). ${ }^{12,13}$ This was one of the highest rates of AD ever reported in a population-based study. An understanding of the risk factors for early $\mathrm{AD}$ in this population may provide a much-needed opportunity for prevention and screening programmes.

\section{Methods}

\section{Sampling procedure}

The Meiktila Eye Study (MES) was a population-based, cross-sectional ophthalmic survey of the inhabitants of rural villages in central Myanmar. The study was conducted within the Mandalay Division, an area encompassing $34253 \mathrm{~km}^{2}$ divided into seven second-order administrative districts of approximately equal size. The township of Meiktila (population approximately 251 000) lies centrally in the Meiktila District and is the only urban area in the region. The district is arbitrarily divided by the Ministry of Health (MOH) into six zones served by a centrally located eye hospital in Meiktila.

Participants were randomly selected using a stratified, cluster sampling process. A sampling frame consisting of a list of all villages in the Meiktila District with their populations was obtained from the $\mathrm{MOH}$. Villages were arbitrarily stratified as large (population $>825$ ) or small (population $\leqslant 825$ ), with small villages in each of the six zones within the Meiktila District constituting six separate strata. For logistical reasons, sampling was restricted to villages within a $3 \mathrm{~h}$ drive from Meiktila (an area encompassing approximately $80 \%$ of the district). All persons 40 years and over within each selected village were eligible for inclusion. The sample size was based on the desired precision of the estimate of blindness (the principal aim of the MES). Health-care workers from Meiktila Township enumerated the selected villages (and advertized and promoted the survey) before commencement of the survey. Six small villages (one from each zone) and four large villages were enumerated, providing a total sample population of 2481 people. All of the participants were self-identified as belonging to the Burman ethnic group.

\section{Data collection}

Data collection took place at the end of the rainy season in November 2005. A single survey team conducted the entire study. Each team member was assigned specific tasks and was well trained in the appropriate area. All equipment and personnel were transported to each village, and the data collection took place on-site. Qualified health-care workers obtained medical and ophthalmic history from each patient in their own language. Each participant then received a comprehensive vision and eye examination.

Visual acuity was tested unaided and with a pinhole using well-illuminated Snellen chart at $6 \mathrm{~m}$, and a non-cycloplegic automatic objective refraction (Humphrey 590 Automatic Refractor; Humphreys Systems, Dublin, CA, USA) was performed. Hand-held ocular biometry (Ocuscan, Alcon Laboratories Inc., Fort Worth, TX, USA) was performed by experienced technicians/ophthalmologists before instilling mydriatics and AL, ACD, and lens thickness were measured for each eye. Each scan was individually interpreted by experienced biometrists and recordings were continued until at least three consistent good quality scans were obtained. A standard deviation $<0.15 \mathrm{~mm}$ for AL was required. These were averaged and recorded; if satisfactory recordings could not be obtained, the data were designated as 'not recordable'. IOP was measured with a Goldmann applanation tonometer (Haag-Streit, Koeniz, Switzerland) and anterior-segment examination was performed at the slit lamp (Haag-Streit). The presence of previous iris ischaemia or pseudoexfoliation was recorded. Two experienced ophthalmologists (RJC and SM) performed the gonioscopy using a Sussman goniolens. Static gonioscopy was performed in dim light with minimal pressure on the cornea, using a short slit beam; each quadrant was graded using a Scheie-type classification. If $>90$ degrees of posterior TM was visible, the pupil was dilated with tropicamide $1 \%$ and phenylephrine $2.5 \%$. Eyes with 'occludable' angles were dilated with tropicamide $0.5 \%$ only and kept under observation for $4 \mathrm{~h}$, or if not possible, they were not dilated. If either eye had evidence of previous AACG (see definition below), then neither eye was dilated.

Two experienced ophthalmologists (HSN and JM) performed the lens gradings after dilation using the Lens Opacification Grading System III. ${ }^{14}$ The agreement between the lens gradings was good (Fleiss $\kappa=0.78$ ).

Optic disc and retinal examinations were then performed using a 78-D lens and reference to standard disc images. The vertical cup/disc ratio (CDR) and the presence of focal notching were recorded. The agreement between the data of the two ophthalmologists was good for grading the angle ( $\left(\right.$ Fleiss $\left.^{\prime} \kappa\right)=0.78$ and determining the CDR $($ Fleiss' $\kappa))=0.72$. 


\section{Ethics}

The MES was approved by the MOH in Myanmar and had ethical approval from the Royal Adelaide Hospital Ethics Committee. Consent for participation was obtained from the head of each village before commencement of the survey. Informed consent, in the participant's own language, was obtained from all participants. The study was conducted in accordance with the Declaration of Helsinki.

\section{Statistics}

Univariate analyses were performed using singlevariable, binary logistic regression (equivalent to $t$-tests for continuous or $\chi^{2}$ tests for categorical data). A multivariate regression model was constructed. To reduce the likelihood of 'overfitting' the model, the ratio of the number of subjects with the condition of interest (the dependent variable) to the number of predictors was $15: 1$, and the predictors were chosen before data exploration, and were in the following order: age, degree of nuclear opalescence (NO), ACD, lens thickness, gender, $\mathrm{AL}$, cortical grade, posterior subcapsular cataract grade, corneal radius, spherical equivalent (SE), and IOP. To preserve information integrity, continuous variables were not categorized. A forced-entry method was used for the multivariate analyses. Residuals were examined for influential cases using Cook's distance and tests (including average variance inflation factor) for the presence of multicollinearity were performed. Statistical analysis was performed using a commercially available statistical software package (SPSS for Windows, version 11.5, SPSS Inc., Chicago, IL, USA). Odds ratios (ORs) with 95\% confidence intervals were calculated for each predictor. A Hosmer and Lemeshow goodness-of-fit and a Nagelkerke $R^{2}$ were calculated. In subjects positive for the dependent variable in both the eyes, the right eye was used for analysis, and in unilateral cases, all variables were drawn from the affected side. A $P$-value of $<0.05$ was considered significant.

\section{Definitions}

The International Society for Geographical and Epidemiological Ophthalmology (ISGEO) has recently proposed definitions, which reserve the term 'glaucoma' for those with glaucomatous optic neuropathy (GON). Those eyes with iridocorneal angles traditionally described as 'occludable' in epidemiological studies ( $<90$ degrees of posterior meshwork visible on gonioscopy) and considered at risk of angle-closure glaucoma have been renamed as primary angle-closure suspects (PACS) and those meeting criteria for PACS, but with peripheral anterior synechiae (PAS) and without GON, are described as having primary angle closure (PAC). Subjects with either PACS or PAC were pooled into a group described as having early AD. The definitions of PACS and PAC were based on the definitions suggested by the ISGEO. If $<90$ degrees of posterior TM were visible with static gonioscopy, the eye was designated as PACS. PAC was defined as any eye with PACS and PAS and/or elevated IOP (>97.5th percentile for the population ( $\geqslant 22 \mathrm{mmHg}$ in this study)) and/or iris whorling or stromal atrophy, but without any GON. PAS was defined as an area of iridotrabecular contact, which could not be broken during dynamic gonioscopy. Surgically induced PAS was not included in this report, but previous intraoclular surgery was not an exclusion criterion.'

Subjects with GON were not included in the analysis. Lens position (LP) was defined as ACD $+1 / 2$ (lens thickness); relative lens position (RLP) was defined as $\mathrm{LP} / \mathrm{AL}$.

\section{Results}

A total of 2481 individuals were eligible and 2076 were examined (836 males, 1240 females: participation rate $83.7 \%$ ). The mean age was 56.2 years; 57 (SD \pm 11.5 ) years for men and $56(S D \pm 11.5)$ years for women. Sufficient examination data to diagnose occludable angles (as defined above) in at least one eye was obtained in 2050 participants (126 were excluded due to unrecordable measurements), 52 had PACG and were excluded; hence, 1998 were included in the analyses. Of these, there were 117 participants with PACS and a further 31 with PAC in at least one eye, giving a total of 148 subjects defined as having occludable angles, but not glaucoma. Table 1 shows the biometric features of the normal compared to the occludable population. Table 2 shows the comparison of biometric features by gender in the normal population and in the occludable population.

Table 1 Biometric measurements in the normal and occludable populations

\begin{tabular}{lccr}
\hline & $\begin{array}{c}\text { Occludable } \\
(\mathrm{n}=148)\end{array}$ & $\begin{array}{c}\text { Normal } \\
(\mathrm{n}=1850)\end{array}$ & P-value \\
\hline AL (mm) (SD) & $21.91(0.86)$ & $22.74(0.93)$ & $<0.001$ \\
ACD (mm) (SD) & $2.60(0.35)$ & $2.84(0.80)$ & $<0.001$ \\
LT (mm) (SD) & $4.54(0.32)$ & $4.46(0.31)$ & 0.002 \\
LP (mm) (SD) & $4.82(0.35)$ & $5.04(0.35)$ & $<0.001$ \\
RLP (mm) (SD) & $0.219(0.015)$ & $0.222(0.016)$ & 0.079 \\
\hline
\end{tabular}

$\mathrm{ACD}=$ anterior chamber depth; $\mathrm{AL}=$ axial length; $\mathrm{LP}=$ lens position $=$ $\mathrm{ACD}+1 / 2(\mathrm{LT}) ; \mathrm{LT}=$ lens thickness; $\mathrm{RLP}=$ relative lens position $=$ $\mathrm{LP} / \mathrm{AL}$. 
Table 2 Biometric measurements by gender

\begin{tabular}{|c|c|c|c|c|c|c|}
\hline & \multicolumn{3}{|c|}{ Normals $(\mathrm{n}=1850)$} & \multicolumn{3}{|c|}{ Occludable $(\mathrm{n}=148)$} \\
\hline & $A L(m m)(S D)$ & $A C D(\mathrm{~mm})(S D)$ & $L T(m m)(S D)$ & $A L(m m)(S D)$ & $A C D(m m)(S D)$ & $L T(m m)(S D)$ \\
\hline Male $(n=805)$ & $23.00(1.0)$ & $2.87(0.45)$ & $4.47(0.32)$ & 22.59 (1.1) & $2.64(0.34)$ & $4.55(0.38)$ \\
\hline Female $(n=1193)$ & $22.50(0.80)$ & $2.80(0.97)$ & $4.45(0.30)$ & $21.70(2.1)$ & $2.58(0.33)$ & $4.57(0.27)$ \\
\hline$P$-value & $<0.001$ & $<0.01$ & 0.18 & 0.01 & 0.38 & 0.80 \\
\hline
\end{tabular}

$\mathrm{ACD}=$ anterior chamber depth; $\mathrm{AL}=$ axial length; $\mathrm{LT}=$ lens thickness.

Table 3 Univariate analysis for occludable angles

\begin{tabular}{|c|c|c|c|c|}
\hline & \multirow[t]{2}{*}{ P-value } & \multirow[t]{2}{*}{ Odds ratio } & \multicolumn{2}{|c|}{$95 \% C I$} \\
\hline & & & Lower & Upper \\
\hline Age (years) & $<0.001$ & 1.038 & 1.024 & 1.052 \\
\hline \multicolumn{5}{|l|}{ Age group } \\
\hline $40-49$ & & 1 & 1 & 1 \\
\hline $50-59$ & 0.001 & 2.37 & 1.42 & 3.94 \\
\hline $60-69$ & $<0.001$ & 2.91 & 1.72 & 4.93 \\
\hline $70+$ & $<0.001$ & 3.96 & 2.34 & 6.69 \\
\hline \multicolumn{5}{|l|}{ Gender } \\
\hline Male & & 1 & 1 & 1 \\
\hline Female & 0.001 & 1.86 & 1.29 & 2.67 \\
\hline NO & $<0.001$ & 1.34 & 1.20 & 1.49 \\
\hline Nuclear cataract & $<0.001$ & 2.32 & 1.58 & 3.39 \\
\hline Cortical grade & 0.004 & 1.23 & 1.07 & 1.41 \\
\hline Cortical cataract & 0.01 & 1.89 & 1.17 & 3.06 \\
\hline PSCC grade & 0.036 & 1.23 & 1.04 & 1.49 \\
\hline PSCC cataract & 0.22 & 1.53 & 0.78 & 3.03 \\
\hline $\mathrm{ACD}(\mathrm{mm})$ & $<0.001$ & 0.11 & 0.06 & 0.21 \\
\hline Lens thickness (LT) (mm) & 0.013 & 2.82 & 1.18 & 4.07 \\
\hline Axial length (AL) (mm) & $<0.001$ & 0.39 & 0.31 & 0.47 \\
\hline Corneal radius (mm) & 0.03 & 0.77 & 0.60 & 0.98 \\
\hline Lens position (LP) & $<0.001$ & 0.19 & 0.11 & 0.32 \\
\hline Relative lens position (RLP) & 0.08 & 0.0 & 0.0 & 3.61 \\
\hline S.E. & 0.047 & 0.96 & 0.92 & 1.00 \\
\hline IOP (mmHg) & 0.004 & 1.04 & 1.01 & 1.08 \\
\hline
\end{tabular}

$\mathrm{PACG}=$ primary angle-closure glaucoma; $\mathrm{NO}=$ nuclear opalescence $\mathrm{ACD}=$ anterior chamber depth; $\mathrm{PSCC}=$ posterior subcapsular cataract S.E. $=$ spherical equivalent; Nuclear cataract $=$ grade $>4.0$, cortical cataract $=$ grade $>2.0, \quad \mathrm{PSCC}=$ grade $>2.0 ; \quad \mathrm{LP}=\mathrm{ACD}+1 / 2 \quad(\mathrm{LT})$; $\mathrm{RLP}=\mathrm{LP} / \mathrm{AL}$

*Odds ratios are given for increasing values of the independent variables, e.g., for each mm reduction in ACD the OR increases ninefold.

Table 3 shows the univariate analysis for occludable angles. Age $(P<0.001)$, female gender $(P<0.001)$, nuclear and cortical cataract $(P<0.001), \operatorname{ACD}(P<0.001)$, lens thickness $(P<0.001)$, AL $(P<0.001)$, corneal radius
$(P=0.03)$, lens position $(P=<0.001)$, SE $(P=0.047)$, and IOP $(P<0.001)$ were all significantly associated with occludable angles.

In the multivariate analysis of occludable angles (Table 4), age, ACD, and AL were all highly significant predictors $(P<0.001$ for each). NO was the only other significant predictor in this model $(P=0.036)$. This model accounted for $25 \%$ of the variance $\left(R^{2}=0.25\right)$. The Hosmer and Lemeshow tests were nonsignificant for the multivariate model, no individual cases were particularly influential (Cook's distances were all $<0.1$ ) and the variance inflation factors for the model did not suggest problems with collinearity.

\section{Discussion}

There is clear evidence from comparisons of populationbased studies that PACG is more common in people of East Asian extraction than in Caucasions or Africans. ${ }^{2-4}$ Univariate analyses from population-based studies have generally confirmed the presence of anatomical risk factors in East Asian eyes ${ }^{4,10,6,8}$; however, multivariate analyses from population-based studies are scarce. This is largely due to the relatively small numbers of subjects with AD (referring to PACS, PAC, and PACG) who are detected. It is only recently that large-scale studies, mainly conducted in Asia, have produced enough subjects with AD to conduct such analyses.

In a recent population-based study from the state of Tamil Nadu in southern India, Vijaya et $a l^{15}$ found, in a univariate analysis, that AL and ACD were significantly shorter in PAC and PACG compared with the normal population; but in a multivariate analysis that included age, they were not. In an earlier report from the same study, in univariate analyses, those with PACS were also found to have shorter ALs, and shallower ACD's, but not thicker lenses than age-matched normals. ${ }^{16}$ Multivariate analyses were not performed in this analysis. Dandona et $a l^{17}$ in the Andhra Pradesh Eye Disease Study reported a multivariate analysis of demographic risk factors for AD. In this analysis, only increasing age was a significant predictor of $\mathrm{AD}$; gender, refractive error, and socioeconomic status were not. ${ }^{17}$ Biometry was not performed in this study. Friedman et al ${ }^{18}$ recently found 
Table 4 Multivariate analysis for occludable angles

\begin{tabular}{|c|c|c|c|c|c|}
\hline & \multirow[t]{2}{*}{$B(S E)$} & \multirow[t]{2}{*}{ P-value } & \multirow[t]{2}{*}{ Odds ratio (OR) } & \multicolumn{2}{|c|}{$95 \%$ CI for $O R$} \\
\hline & & & & Lower & Upper \\
\hline Age (years) & $0.05(0.01)$ & $<0.001$ & 1.06 & 1.03 & 1.08 \\
\hline $\mathrm{AL}$ & $-0.93(.16)$ & $<0.001$ & 0.40 & 0.29 & 0.54 \\
\hline $\mathrm{ACD}$ & $-1.69(0.42)$ & $<0.001$ & 0.18 & 0.08 & 0.42 \\
\hline Lens thickness & $0.55(0.42)$ & 0.16 & 1.73 & 0.80 & 3.74 \\
\hline \multicolumn{6}{|l|}{ Gender } \\
\hline Male & & & 1 & 1 & 1 \\
\hline Female & $0.36(0.29)$ & 0.21 & 1.43 & 0.82 & 2.50 \\
\hline $\mathrm{NO}$ & $0.20(0.10)$ & 0.036 & 1.23 & 1.01 & 1.48 \\
\hline Cortical grade & $1.00(0.16)$ & 0.54 & 1.10 & 0.81 & 1.51 \\
\hline PSCC grade & $0.09(0.16)$ & 0.57 & 1.10 & 0.80 & 1.51 \\
\hline Corneal radius & $0.04(0.18)$ & 0.81 & 1.04 & 0.73 & 1.48 \\
\hline S.E. & $-0.01(0.03)$ & 0.73 & 0.99 & 0.94 & 1.05 \\
\hline IOP $(\mathrm{mmHg})$ & $0.004(0.032)$ & 0.91 & 1.00 & 0.94 & 1.07 \\
\hline
\end{tabular}

$\mathrm{NO}=$ nuclear opalescence; $\mathrm{ACD}=$ anterior chamber depth; $\mathrm{PSCC}=$ posterior subcapsular cataract; $\mathrm{AL}=$ axial length; $\mathrm{S} . \mathrm{E}$. = spherical equivalent; Model constant $=3.78$; Nagelkerke $R^{2}=0.25$; Hosmer and Lemeshow test $=0.938$; Average variance inflation factor $=1.2$.

that contralateral eyes of individuals having had an AACG attack tend to be shorter and have more crowded anterior segments than those of healthy controls. They found that after adjusting for age and sex, these eyes had a mean adjusted AL that was $1.2 \mathrm{~mm}$ shorter, an optical ACD that was $0.63 \mathrm{~mm}$ shallower $(24 \%$ shallower than controls), and lenses that were, on average, $0.35 \mathrm{~mm}$ thicker $(P<0.01) .^{18}$

In this study population, women with non-occludable angles had significantly shorter ACDs than men with non-occludable angles. However, in women with occludable angles the difference in ACD compared to men with occludable angles was not significant. This reinforces the notion that female gender as a risk factor for PACG is related to the generally shorter ACD in women, but men with shallow ACDs are at similar risk.

In this study, occludable angles were significantly associated with all the demographic and biometric variables tested, including female gender, age, ACD, AL, and lens opacities. Those with occludable angles tended to have a more anterior lens position; however, RLP was not significantly associated. Similar findings were recently reported in a study comparing eyes affected by acute angle-closure with the fellow eye. ${ }^{19}$ Previous studies have reported an association with hypermetropia ${ }^{20,21}$; the converse was found in this population, but is likely to be due to nuclear cataractassociated lenticular myopia. ${ }^{6}$ Clearly, many of these variables are correlated, especially age and lens opacity, and in the multivariate analysis the most significant anatomical predictors of occludability were ACD and AL. Age also remained significant, as did NO, but gender was not significant in this model. IOP was significantly

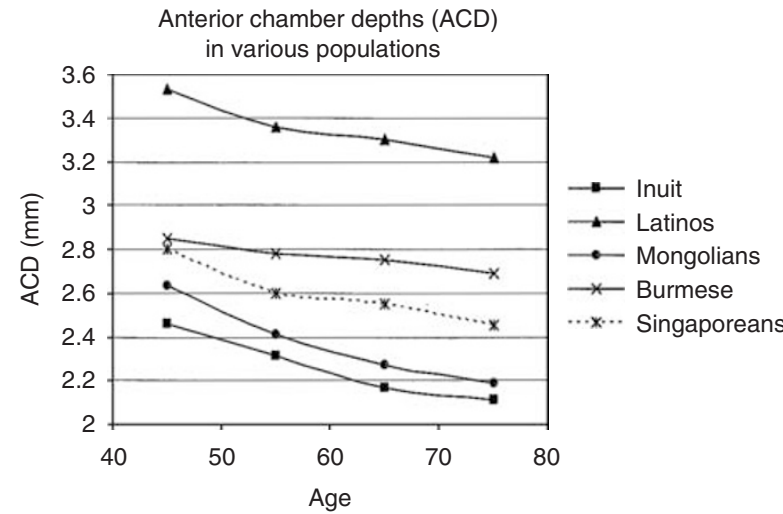

Figure 1 Anterior chamber depths (ACD) in various populations. ACD decreases with age in all populations and is shallowest in the Inuit ${ }^{2}$ and deepest in the Caucasian Latino population. ${ }^{25}$ (Data has been assimilated as an average of agespecific ACD and adapted from Johnson and Foster. ${ }^{26}$

associated in the univariate analysis but not the multivariate (Figure 1).

ACD had the highest OR, such that for every $1 \mathrm{~mm}$ reduction in ACD there was a 5.6 times increased likelihood of occludability. The ACD reduces with age due to increasing lens thickness; however, the former remained an independent predictor of occludability, whereas the latter did not. Although lens thickness is theoretically a risk factor for occludability, studies in southern India and South Africa have also found no difference between lens thickness in those with PACG and normals. ${ }^{15,21}$ It is possible that axial lens thickness measurements do not closely correlate with the peripheral lens thickness. In a clinical series from 
Singapore, Lim et $a l^{19}$ found that compared with fellow eyes, AACG-affected eyes had significantly shallower ACDs, more anterior lens position, and less cortical cataract. There was no significant difference in the degree of nuclear cataract. ${ }^{10}$ However, earlier studies have reported that eyes with AACG tend to have thicker lenses than age-matched normal eyes. ${ }^{22,23}$

The significant association of nuclear cataract with occludable angles, which persisted in the multivariate analysis was particularly interesting. A possible explanation for the association of occludability with $\mathrm{NO}$ may be that with increasing NO there may be an increase in the anterior curvature of the lens. Lowe ${ }^{24}$ reported this biological parameter as one of the strongest associations with $\mathrm{AD}$; however, to our knowledge, the relationship between NO and lens curvature has not been reported. An alternative explanation could be an inversion of cause and effect, with occludable angles precipitating nuclear lens opacity.

In conclusion, in this Burmese population, those with early AD had significantly shorter ALs, ACD's and thicker lenses than those without occludable angles. In univariate analyses, nuclear and cortical cataract, increasing age, female gender, steeper corneal curvature and decreasing minus SE were also significantly associated with occludability. However, in multivariate analyses, increasing age, decreasing $\mathrm{AL}$, decreasing $\mathrm{ACD}$ and nuclear cataract were the significant predictors of early AD. To our knowledge cataract per se has not previously been described as an independent risk factor for AD. The presence of nuclear cataract should raise the clinical suspicion of the possibility of $\mathrm{AD}$ in this population, which should be confirmed by gonioscopy.

\section{Acknowledgements}

Financial support was provided by an Independent Grant Scheme from Pfizer Australia. There are no commercial conflicts.

\section{References}

1 Resnikoff S, Pascolini D, Etya'ale D, Kocur I, Pararajasegaram R, Pokharel GP et al. Global data on visual impairment in the year 2002. Bull World Health Organ 2004; 82(11): 844-851.

2 Alsbirk PH. Primary angle-closure glaucoma. Oculometry, epidemiology, and genetics in a high risk population. Acta Ophthalmol Suppl 1976; 127: 5-31.

3 Salmon JF, Mermoud A, Ivey A, Swanevelder SA, Hoffman $\mathrm{M}$. The prevalence of primary angle closure glaucoma and open angle glaucoma in Mamre, western Cape, South Africa. Arch Ophthalmol 1993; 111(9): 1263-1269.

4 Foster PJ, Baasanhu J, Alsbirk PH, Munkhbayar D, Uranchimeg D, Johnson GJ. Glaucoma in Mongolia. A population-based survey in Hovsgol province, northern Mongolia. Arch Ophthalmol 1996; 114(10): 1235-1241.

5 Foster PJ. The epidemiology of primary angle closure and associated glaucomatous optic neuropathy. Semin Ophthalmol 2002; 17(2): 50-58.

6 Salmon JF. Predisposing factors for chronic angle-closure glaucoma. Prog Retin Eye Res 1999; 18(1): 121-132.

7 Foster PJ, Devereux JG, Alsbirk PH, Lee PS, Uranchimeg D, Machin D et al. Detection of gonioscopically occludable angles and primary angle closure glaucoma by estimation of limbal chamber depth in Asians: modified grading scheme. Br J Ophthalmol 2000; 84(2): 186-192.

8 Aung T, Nolan WP, Machin D, Seah SK, Baasanhu J, Khaw PT et al. Anterior chamber depth and the risk of primary angle closure in 2 East Asian populations. Arch Ophthalmol 2005; 123(4): 527-532.

9 Congdon NG, Youlin Q, Quigley H, Hung PT, Wang TH, Ho $\mathrm{TC}$ et al. Biometry and primary angle-closure glaucoma among Chinese, white, and black populations. Ophthalmology 1997; 104(9): 1489-1495.

10 He M, Foster PJ, Johnson GJ, Khaw PT. Angle-closure glaucoma in East Asian and European people. Different diseases? Eye 2006; 20(1): 3-12.

11 Thomas R, George R, Parikh R, Muliyil J, Jacob A. Five year risk of progression of primary angle closure suspects to primary angle closure: a population based study. $\mathrm{Br} \mathrm{J}$ Ophthalmol 2003; 87(4): 450-454.

12 Casson RJ, Newland HS, Muecke J, McGovern S, Durkin S, Sullivan Tet al. Prevalence and causes of visual impairment in rural Myanmar: the Meiktila Eye Study. Ophthalmology 2007; 114(12): 2302-2308.

13 Casson RJ, Newland HS, Muecke J, McGovern S, Abraham $\mathrm{L}$, Shein WK et al. Prevalence of glaucoma in rural Myanmar: the Meiktila Eye Study. Br J Ophthalmol 2007; 91(6): 710-714.

14 Chylack Jr LT, Wolfe JK, Singer DM, Leske MC, Bullimore MA, Bailey IL et al. The Lens Opacities Classification System III. The Longitudinal Study of Cataract Study Group. Arch Ophthalmol 1993; 111(6): 831-836.

15 Vijaya L, George R, Arvind H, Baskaran M, Paul PG, Ramesh SV et al. Prevalence of angle-closure disease in a rural southern Indian population. Arch Ophthalmol 2006; 124(3): 403-409.

16 George R, Paul PG, Baskaran M, Ramesh SV, Raju P, Arvind $\mathrm{H}$ et al. Ocular biometry in occludable angles and angle closure glaucoma: a population based survey. $\mathrm{Br} \mathrm{J}$ Ophthalmol 2003; 87(4): 399-402.

17 Dandona L, Dandona R, Mandal P, Srinivas M, John RK, McCarty CA et al. Angle-closure glaucoma in an urban population in southern India. The Andhra Pradesh eye disease study. Ophthalmology 2000; 107(9): 1710-1716.

18 Congdon NG, Friedman DS. Angle-closure glaucoma: impact, etiology, diagnosis, and treatment. Curr Opin Ophthalmol 2003; 14(2): 70-73.

19 Lim MC, Lim LS, Gazzard G, Husain R, Chan YH, Seah SK et al. Lens opacity, thickness, and position in subjects with acute primary angle closure. J Glaucoma 2006; 15(3): 260-263.

20 Lowe RF. Aetiology of the anatomical basis for primary angle-closure glaucoma. Biometrical comparisons between normal eyes and eyes with primary angle-closure glaucoma. Br J Ophthalmol 1970; 54(3): 161-169.

21 Salmon JF. Presenting features of primary angle-closure glaucoma in patients of mixed ethnic background. S Afr Med J 1993; 83(8): 594-597. 
22 Lowe RF. Causes of shallow anterior chamber in primary angle-closure glaucoma. Ultrasonic biometry of normal and angle-closure glaucoma eyes. Am J Ophthalmol 1969; 67(1): 87-93.

23 Luntz MH. Primary angle-closure glaucoma in urbanized South African caucasoid and negroid communities. $\mathrm{Br} \mathrm{J}$ Ophthalmol 1973; 57(7): 445-456.

24 Lowe RF. Anterior lens curvature. Comparisons between normal eyes and those with primary angle-closure glaucoma. Br J Ophthalmol 1972; 56(5): 409-413.

25 Shufelt C, Fraser-Bell S, Ying-Lai M, Torres M, Varma R. Refractive error, ocular biometry, and lens opalescence in an adult population: the Los Angeles Latino Eye Study. Invest Ophthalmol Vis Sci 2005; 46(12): 4450-4460.

26 Johnson GJ, Foster PJ. Can we prevent angle-closure glaucoma? Eye 2005; 19(10): 1119-1124. 